\title{
http://dx.doi.org/10.35381/r.k.v5i1.803
}

\section{Aplicaciones tecnológicas y motricidad fina en niños de 3 a 6 años}

Technological applications and fine motor skills in children from 3 to 6 years old

\author{
Javier Esteban Zumba-Villavicencio \\ javier.zumba@psg.ucacue.edu.com \\ Universidad Católica de Cuenca, Azogues \\ Ecuador \\ https://orcid.org/0000-0003-3092-766X \\ Darwin Gabriel Garcia-Herrera \\ dggarciah@ucacue.edu.ec \\ Universidad Católica de Cuenca, Azogues \\ Ecuador \\ https://orcid.org/0000-0001-6813-8100 \\ Cristián Andrés Erazo-Álvarez \\ cristianerazo@ucacue.edu.ec \\ Universidad Católica de Cuenca, Cuenca \\ Ecuador \\ https://orcid.org/0000-0001-8746-4788 \\ Juan Carlos Erazo-Álvarez \\ jcerazo@ucacue.edu.ec \\ Universidad Católica de Cuenca, Cuenca \\ Ecuador \\ https://orcid.org/0000-0001-6480-2270
}

Recibido: 27 de abril de 2020

Revisado: 30 de mayo de 2020

Aprobado: 16 de junio de 2020

Publicado: 30 de junio de 2020 


\title{
RESUMEN
}

La educación, tecnología y psicomotricidad son un desafío para los docentes, sin embargo, en la actualidad se han hecho grandes esfuerzos para compaginar varios ámbitos de la educación y la tecnología a través de la capacitación docente. El objetivo de la investigación es analizar la relación las competencias docentes relacionadas a la tecnología y la motricidad fina, para estos se utilizó una investigación descriptiva, no experimental de cohorte transversal. Dentro de los resultados evidenciados es que el 70,9\% de los docentes tienen una escasez de conocimientos de tecnología, a pesar de conocer su importancia, se concluye que las competencias tecnológicas docentes de educación inicial, no se orientan primordialmente al desarrollo motriz de los niños si no a otros aspectos relacionados con conocimientos teóricos.

Descriptores: Experimento educacional; innovación educacional; planificación de la educación; tecnología de la información. (Palabras tomadas del Tesauro UNESCO).

\begin{abstract}
Education, technology and psychomotricity are a challenge for teachers, however, great efforts have been made at present to combine various areas of education and technology through teacher training. The objective of the research is to analyze the relationship between teaching skills related to technology and fine motor skills. For these, a descriptive, non-experimental cross-cohort investigation was used. Among the results evidenced is that $70.9 \%$ of teachers have a shortage of technology knowledge, despite knowing its importance, it is concluded that the technological skills of initial education teachers are not primarily oriented to the motor development of children if not other aspects related to theoretical knowledge.
\end{abstract}

Descriptors: Educational experiments; educational innovations; educational planning; information technology. (Words taken from the UNESCO Thesaurus). 


\section{INTRODUCCIÓN}

En el presente trabajo se pretende realizar una explicación del desarrollo de la motricidad fina en niños con una implicación de la tecnología para la estimulación de dicha área. Para ello inicialmente, se hace un recorrido de los conceptos básicos relacionados; luego se hace énfasis en la aplicación práctica y profesional desde distintos puntos de vista y desde distintos resultados tras la corroboración de evidencia empírica, con la finalidad última de conformar un cuerpo teórico concreto y específico sobre la motricidad fina que pueda ser objeto de práctica docente. La investigación tiene como objetivo general analizar varias bases conceptuales y teóricas relacionadas a la motricidad fina y su desarrollo mediante el uso de la tecnología.

El problema de esta investigación se sitúa que a nivel del Ecuador la implementación tecnológica como parte de un proceso de aprendizaje no está estrechamente vinculado. A pesar de que el Ministerio de Educación del Ecuador dispone lineamientos curriculares de informática aplicada a la educación en los cuales consta la recomendación de que en el subnivel de preparatoria se brinde una hora pedagógica semanal; y del mismo modo en el subnivel elemental dos horas pedagógicas semanales. De este modo, se puede inferir que la educación en tecnología es poco desarrollada en el ámbito pedagógico. Bajo este mismo enfoque, surge la necesidad de introducir herramientas tecnológicas que posean características particulares según la etapa evolutiva del individuo y su desarrollo cognoscitivo.

A partir de la necesidad ante evidenciada se establece la importancia de iniciar el proceso de desarrollo psicomotriz en las edades de 3 a 6 años. Según el modelo de desarrollo motor de (Berruezo-Adelantado, 2008) se muestra que a partir de los dos años hasta los siete años los niños se encuentran en la etapa de adquisición de habilidades básicas, misma que se divide en tres estadios: estadio inicial, elemental y maduro. Sin embargo, para realizar el análisis se ha toma el rango etario de 3 a 6 años debido a que a los tres años lo niños inician su proceso de escolarización y a los seis años se da la consolidación del proceso lecto-escritor.

La presente investigación abarca temas sobre la perspectiva tecnológica en la motricidad fina en el entorno educativo, utilizando como técnica de enseñanza la tecnología, puesto que actualmente hay un desconocimiento sobre la utilización de la 
tecnología para desarrollar la motricidad fina en los niños. Se realizó un estudio previo a diferentes profesores, para establecer el nivel de conocimiento y la aplicación de tecnológica en el desarrollo de sus clases.

La tecnología es una de las artes más actuales y necesaria para el desarrollo de la civilización, por lo cual vemos necesaria la utilización de la misma en las aulas clases (Quevedo-Álava, et al., 2020), también tiene una presencia importante en el desarrollo motriz, donde los niños aprenden jugando y qué mejor forma de hacerlo, que, utilizando tecnología innovadora, si nos damos a entender los niños nacen con un conocimiento tecnológico y no son migrantes tecnológicos, por lo que se les hace más fácil el aprendizaje nuevo. La presente investigación tiene el objetivo de incluir la tecnología en el aula clases para que los docentes desarrollen la psicomotricidad de sus alumnos.

\section{Referencial teórico}

\section{Psicomotricidad}

Para adentrarnos en el tema es necesario abordar algunos términos que se debe mencionar y diferenciar, es la psicomotricidad y la motricidad. La psicomotricidad según (Tapia-Camargo, Azaña-Estrella, \& Tito-Córdova, 2014) "es el desarrollo físico, psíquico e intelectual que se produce en el sujeto a través del movimiento" (p. 65). Por lo tanto, se entiende al movimiento como una fuente de maduración fisiológica y psicológica que influye en el desarrollo de la inteligencia y de las funciones cognitivas, así como también el establecimiento de relaciones con el medio ambiente. En este sentido, se ha implementado el término psicomotricidad educativa que hace referencia a la forma de entender la educación bajo dos perspectivas: la psicología evolutiva y la pedagogía activa (Rivas, 2008).

Bajo este enfoque educativo la estimulación motriz fina comúnmente se da por técnicas grafomotrices en las que se intenta perfeccionar la pinza motriz por medio de actividades como: rasgado, hilvanado, trazos, arrugado, cortado, estampado, etc. Con las estrategias para el desarrollo grafomotríz se manejan técnicas para mejorar las destrezas en las manos, dedos y coordinación viso-manual (Vaccaro et al., 2018). Sin 
embargo, esta perspectiva de estimulación motriz no es concordante con una generación que responda a las cuestiones tecnológicas del nuevo milenio.

La psicomotricidad a más de tener un enfoque educativo, también se reconoce como una disciplina reeducativa y terapeuta. Con esta perspectiva se opera como una unidad psicosomática que actúa por medio del cuerpo y el movimiento. Sin embargo, para (Le-Boulch, 1985) constituye una realidad psicofisiológica que permite la adaptación flexible y armoniosa. Sin embargo, el uso de la palabra motricidad se ha estudiado desde teorías amplias que imponen el prefijo psico, existiendo una redundancia conceptual en la que se sigue anclando profundamente el concepto dualista del cuerpo (Castañer \& Camerino, 2014).

Para contextualiza la psicomotricidad es necesario entender el potencial cognoscitivo de la actividad motriz, misma que se ha explicado desde la perspectiva de Jean Piaget con el postulado de equilibración cognoscitiva el cual se manifiesta a través de dos procesos: asimilación como la adquisición de nuevas acciones al bagaje motor ya disponibles; la acomodación como el proceso que ayuda en la adecuación de nuevas acciones dentro de las que ya se poseía antes (Lacasa, 1984). En la actualidad, el desarrollo cognoscitivo es fruto de la combinación de la maduración fisiológica y el aprendizaje; para lo cual se hace la relación causal de: a mayor maduración y mayor aprendizaje es igual a una buena adaptación; por el contrario, sin maduración y sin aprendizaje no existe efecto alguno (Castañer \& Camerino 2006).

\section{Motricidad}

El estudio de la motricidad se diferencia en dos tipos: motricidad gruesa y motricidad fina. En el primer caso hace referencia a acciones grades grupos musculares, los cambios de posición del cuerpo y la capacidad de mantener el equilibrio. Por el contrario, la motricidad fina es la acción de pequeños grupos musculares que se logra a partir de actividades que requieren coordinación y precisión de los músculos cortos de las manos y los dedos (Pentón-Hernández \& Piñeda-Sierra, 2018). En su revisión manifiesta que su desarrollo se da desde los primeros estadios del desarrollo infantil, pero se consolida hasta los ocho años debido a la maduración morfológica y funcional. 
Autores como: (Castañer \& Camerino, 2006) manifiestan que el abordaje de los componentes y manifestaciones de la motricidad human, está dando claves para entender nuestro propio cuerpo y, en consecuencia, proponen el modelo sistémico, en el cual se observa al individuo como una unidad global, organizada e interrelacionar. Entonces, en este contexto se comprende a la motricidad como una pieza clave para el aprendizaje.

En el ámbito de las conductas motrices es inherente la importancia que juega la capacidad cerebral de plasticidad y de ductilidad motriz en cualquier etapa del desarrollo evolutivo de la persona, tomando en cuenta que las magnitudes de los pilares de la motricidad y de la inteligencia se reducen o se amplían respectivamente. El desarrollo temprano de la pinza motora se considera el primer paso para adentrarse a la motricidad fina y reflejo de una maduración cognoscitiva según su edad. En relación al desarrollo del lenguaje, la pinza madura se acompaña de la imitación simbólica (Bates, 1999).

\section{Educación tecnológica}

Las tecnologías cada vez están más presentes en nuestra sociedad y no hablar de ellas es alejarnos de una realidad que forma parte de nuestra cotidianidad. Por ello la educación en tecnología puede estar sujeta al hecho de que el niño lo considere una normalidad dado que conviven con ellas y las adoptan sin mayor dificultad (RomeroTena, 2016). Enseñar por medio de herramientas tecnológicas supone un gran desafío para el personal docente dado que no existe capacitación o formación certificada en el área.

El dominio de la tecnología dentro de la pedagogía supone un manejo dentro de los cuatro pilares fundamentales de la educación que es: aprender a conocer, aprender a hacer, aprender a vivir juntos y aprender a ser; ya que la enseñanza escolar requiere trascender el aprender a conocer, al aprender a hacer desafiando el mundo práctico en una interrelación constante de los conocimientos y relaciones interpersonales; y conjugando con el interés propio del individuo (Delors, 2013). En contraste, la relación entre la educación científica y la educación tecnológica inicialmente estuvo controlada 
por la percepción que se tiene de la relación entre actividades de la ciencia y la tecnología.

La exposición tecnológica se ha visto influenciado por el desarrollo y la diversificación creciente de la educación abierta y a distancia; la rápida inclusión de las diversas tecnologías en el medio educativo con los años ha sido eficiente en relación a los costos, debido a que se puede acoger a gran número de estudiantes que los sistemas educativos convencionales no abarcarían. Los medios tecnológicos según (GallegoLema et al., 2017) es una forma genérica de comunicación asociada con formas particulares de presentar el conocimiento. Cada medio tiene su propia forma de presentar su conocimiento, de organizarlo, su estilo, su formato y su estructura. La tecnología lo que hace es adaptarse estos medios en diferentes necesidades y niveles para que el niño tenga un aprendizaje progresivo.

Para (Luján Ferrer and Salas Madriz 2011) en educación, los cinco medios más utilizados son: contacto directo humano, textos, audio, televisión y computación. De esta forma se evidencia que los criterios generalmente aprobados para la selección de medios tecnológicos en educación se consideran fuera de la innovación académica. Entonces, de acuerdo a los modelos teóricos para la selección de medios tecnológicos se sugiere las siguientes características, cabe mencionar que se puede flexibilizar en relación a las necesidades: "que funcione en una amplia variedad de contextos; que permita tomar decisiones a nivel estratégico o institucional y a nivel táctico o educativo; que atienda de manera equitativa cuestiones educativas y operacionales; que identifique diferencias decisivas entre tecnologías distintas" (Bates, 1999, p. 38).

Una de las condicionantes complejas que tiene la tecnología es el acceso a ella, y esta arista se discute mucho las desigualdades sociales existentes en cada estado, es por ello para estudiantes que no tienen acceso una opción es hacer que la tecnología esté disponible en los centros de estudio. 


\section{Teorías de aprendizaje}

\section{Conductismo}

El conductismo centra su enseñanza en la idea básica de estímulo-respuesta, por lo tanto, niega totalmente las funciones de las estrategias conscientes o de voluntad propia para el aprendizaje. El aprendizaje se sitúa en el marco de recompensas y castigos que viene de medio ambiental. En este caso el trabajo del maestro es programar y organizar una serie de actividades para obtener resultados deseados 0 más apropiados. La tecnología con este enfoque es asociada al análisis y modificaciones de la conducta a través de una enseñanza programada (Ferrer \& Salas-Madriz, 2009).

\section{Teorías cognoscitivas}

En relación a esta teoría que expresa el aprendizaje como resultado de una compleja interacción de las funciones ejecutivas de orden superior e inferior dentro del aprendizaje, así como también resaltan la importancia del aprendizaje de estrategias intelectuales conscientes. Desde esta perspectiva, (Bates, 1999) que un área de trabajo no es definida tan solo por sus contenidos u tópicos, sino que también intervienen los métodos empleados para definir y validar el conocimiento dentro de esta área.

\section{Tecnología y motricidad}

En estudios en los que relacionan la tecnología y la deficiencia motriz revelan que las personas que poseen algún tipo de disminución motriz o la falta de estimulación dentro de los periodos sensibles del desarrollo se encuentran numerosos obstáculos y barreras que les impiden el desarrollo de habilidades, ejecución de actividades, la relación con las personas y el entorno, etc. (Escoin, 2001) a partir de las derivaciones que se obtienen con la disminución motriz se ha implementado recursos tecnológicos e informáticos para identificar la posible mejoría con el uso de la tecnología. Se registra que con implementos tecnológicos que tengan teclado o una funcionalidad en la que se use la pinza motora facilita la estimulación de la motricidad fina (Riva et al., 2014), 
del mismo nodo, con implementos tecnológicos amplios o con directrices de movimiento corporal entero se puede trabajar la motricidad gruesa.

\section{MÉTODO}

Para la realización del presente artículo la metodología aplicada parte del enfoque cuantitativo con un alcance descriptivo (Bernal-Ávila, Erazo-Álvarez, \& NarváezZurita, 2019). La recolección de datos se realizó por medio de la aplicación de una entrevista estructurada, la misma que contenía siete preguntas dirigidas a docentes. Se aplicaron 55 encuestas estructuradas vía online. Dentro de los criterios de inclusión constan los siguientes: que sean docentes del nivel educativo primaria elemental, que actualmente se encuentren laborando y que posean título profesional de docentes.

Para los criterios de inclusión se tomó en cuenta lo siguiente: docentes que laboren en niveles educativos diferentes al nivel educativo elemental y personas que laboren como docentes a pesar de que su título profesional no corresponda. El instrumento investigativo aplicado fue analizado a través del cálculo del coeficiente Alfa de Cronbach, cuyo valor fue de 0,798.

Para el procesamiento de los datos se utilizó el software estadístico SPSS, por lo que, se buscó el resultado de medidas de tendencia central, frecuencias, relación de variable e indicadores de fiabilidad de la información. Con la información tabulada y analizada se procedió a realizar un informe de resultados preliminar (Erazo \& Narváez, 2020).

\section{RESULTADOS}

\section{Tabla 1}

Análisis de frecuencia de datos: Su visión de la tecnología en el desarrollo de la psicomotricidad

\begin{tabular}{lcccc} 
Escala & Frecuencia & Porcentaje & $\begin{array}{c}\text { Porcentaje } \\
\text { válido }\end{array}$ & $\begin{array}{c}\text { Porcentaje } \\
\text { acumulado }\end{array}$ \\
\hline Amplio & 12 & 21,8 & 21,8 & 21,8 \\
Escaso & 39 & 70,9 & 70,9 & 92,7 \\
Nulo & 4 & 7,3 & 7,3 & 100,0 \\
Total & 55 & 100,0 & 100,0 & \\
\hline
\end{tabular}

Fuente: Elaboración propia 
Como se evidencia, la visión que tienen los docentes sobre la tecnología y el desarrollo de la psicomotricidad, de acuerdo a sus respuestas podemos observar que $70,9 \%$ de los docentes no tiene una visión clara de la tecnología y psicomotricidad. Del mismo modo, esto se podría relacionar a la siguiente pregunta que es "Cree que la psicomotricidad es importante para el desarrollo infantil" a lo que un $98,2 \%$ responde de forma positiva. Entonces, a partir de estas respuestas se presume que los docentes conocen la importancia que tiene el desarrollo psicomotriz, pero que no manejan herramientas tecnológicas ya sea para estimularlo o adquirirlo.

En este mismo sentido, en relación a la pregunta sobre si la tecnología ayuda en el desarrollo psicomotriz del niño, el 67,27\% responde de forma afirmativa, pero el 21, $81 \%$ manifiesta desconocer el tema y el 10,91\% responde de forma negativa. Para entender, la relación entre la visión de la tecnología y su opinión sobre su importancia en el desarrollo psicomotriz, se realizó un cruce de variables. (Tabla 2)

\section{Tabla 2}

Estadísticos descriptivos

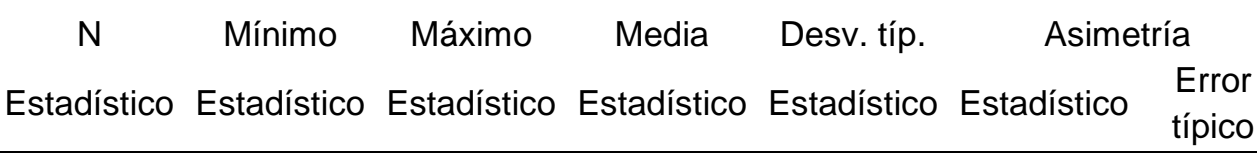

\begin{tabular}{|c|c|c|c|c|c|c|c|}
\hline \multicolumn{8}{|l|}{ La tecnología } \\
\hline $\begin{array}{l}\text { desarrollo } \\
\text { psicomotriz del } \\
\text { niño }\end{array}$ & 55 & 1 & 3 & 1,55 & 0,835 & 1,040 & 0,322 \\
\hline $\begin{array}{l}\text { Su visión de la } \\
\text { tecnología en el }\end{array}$ & & & & & & & \\
\hline $\begin{array}{l}\text { desarrollo de la } \\
\text { psicomotricidad } \\
\text { es }\end{array}$ & 55 & 1 & 3 & 1,85 & 0,524 &,- 181 & 0,322 \\
\hline $\begin{array}{l}\mathrm{N} \text { válido (según } \\
\text { lista) }\end{array}$ & 55 & & & & & & \\
\hline
\end{tabular}

Fuente: Elaboración propia 
Con respecto a la tabla se puede observar que existe una diferencia significativa entre las dos variables analizadas. Por lo que se muestra que existe una asimetría positiva y una asimetría negativa, dando como resultado una poca consistencia de los datos en cuanto a su distribución respecto a la media aritmética. Los intervalos de confianza para identificar una diferencia en las dos variables oscilan en el 0,6 entre las dos variables analizadas.

Los resultados evidenciados sobre el conocimiento de los docentes en tecnología aplicada en la psicomotricidad y pedagogía para niños son escasos, puesto que su interés no contempla la tecnología como un medio de aprendizaje dado el desconocimiento y la falta de recursos que subyacen. En este sentido, Roig concluye que las prácticas de enseñanza son complejas y que está complejidad no pude analizarse con metodologías que lo simplifiquen. Es por ello que la formación docente no responde a las necesidades dada la complejidad de su aplicación; con esto no se quiere obviar su importancia dentro de la educación.

La formación docente y el conocimiento didáctico tecnológico supone una determinada posición en el campo pedagógico y estructura social, para ello se muestra que el interés docente relacionado a las innovaciones tecnológicas pasa desapercibido. Por ello, (Gewerc-Baruje, Pernas-Morado, \& Varela-Pet, 2013) manifiesta, en definitiva, las prácticas de formación que potencien el desarrollo de la dimensión intelectual de la docencia y la adquisición de capacidades de reflexión y análisis, conducirán a una mayor comprensión de la propia práctica docente y del contexto científico, social y cultural en el que se desarrolla.

Uno de los objetivos que se planteo fue detectar el conocimiento tecnológico, pedagógico y disciplinario de los docentes en la integración educativa de las TIC en el desarrollo y estimulación de la psicomotricidad, de forma específica, sobre la motricidad fina. Al respecto, los resultados generales obtenidos indican que el profesorado autopercibe un escaso dominio de sus conocimientos pedagógicos y disciplinares que tecnológicos, confirmando así resultados de otros estudios realizados en el contexto nacional sobre el dominio de conocimientos tecnológicos por parte de los docentes de primaria (Roig-Vila \& Flores-Lueg, 2014). 
El docente es el responsable de diseñar tanto las oportunidades de aprendizaje como el entorno propicio que facilite el uso de las TIC por parte de los estudiantes. Es por este motivo que es fundamental que todos los docentes estén preparados para ofrecer esas oportunidades a sus estudiantes (UNESCO, 2008), porque, a pesar del avance tecnológico, (Cabero Almenara, 2007) nos advierten que en las aulas virtuales se están reproduciendo las metodologías tradicionales que se llevan a cabo en las clases presenciales, utilizando las TIC como recursos de control institucional de los estudiantes, más no de estimulación del mismo.

\section{PROPUESTA}

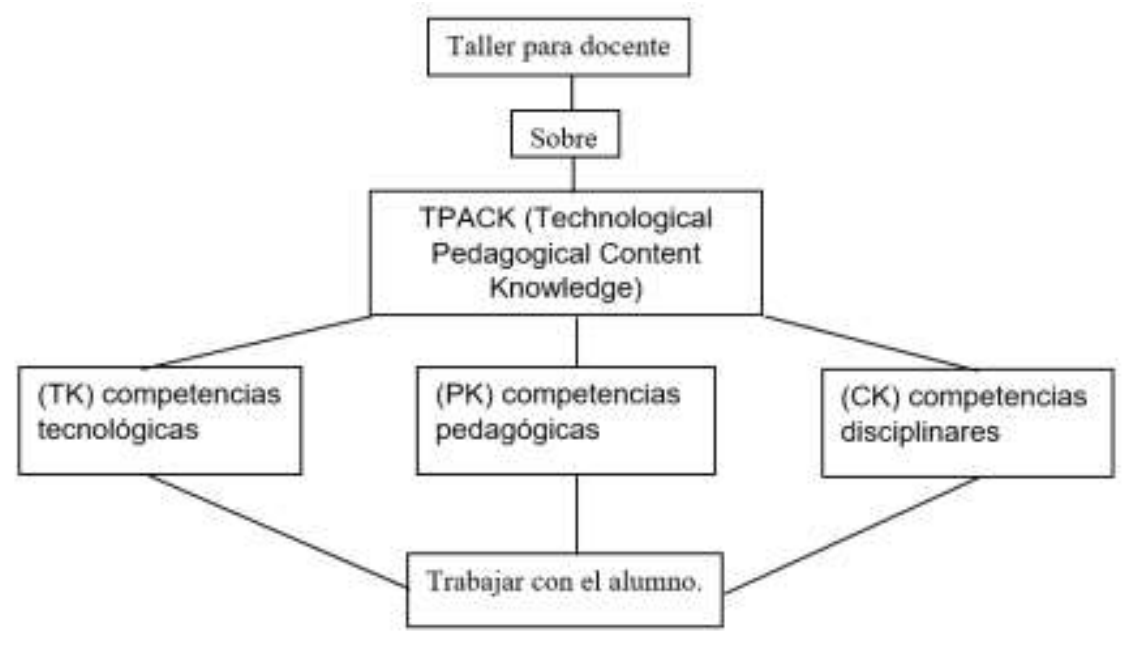

Figura 1. Desarrollo de la motricidad fina a través de tecnología. Fuente: Elaboración propia

\section{Objetivos}

El programa para el desarrollo motriz fino presenta los siguientes objetivos.

1. Familiarizar a los niños con las TIC, en concreto con el IPAD. El primer día se les enseñó a encenderlo y su funcionamiento, así como el acceso a las aplicaciones.

2. Mejorar la motricidad fina en los niños, mediante movimientos principalmente de manos y dedos gracias a la pantalla táctil del IPAD o Tablet. 
3. Agilizar las tareas en los niños relacionadas con la motricidad fina; en concreto abrochar y desabrochar botones, el uso de los cubiertos, superar las dificultades en dibujo y escritura.

\section{Metodología de aplicación}

Modelo de base: el modelo TPACK (Technological Pedagogical Content Knowledge).

\section{Tabla 3}

\section{Dimensiones TPACK}

\section{Dimensiones TPACK}

TK. Competencias tecnológicas

PK. Competencias pedagógicas

CK. Competencias disciplinares

TPK. Competencias tecnológicas pedagógicas.

TCK. Competencias tecnológicas del contenido.

PCK. Competencias pedagógicas del contenido.

TPACK. Competencias tecnológicas y pedagógicas del contenido.

\section{Descripción}

Conocimiento sobre capacidades y aplicaciones tecnológicas. Competencias pedagógicas en general.

Competencias sobre la materia en la que es experto.

Conocimientos que incluyen aspectos tecnológicos y pedagógicos.

Conocimiento sobre cómo la tecnología puede utilizarse para representar la materia a utilizar y desarrollar la competencia disciplinar.

Conocimientos pedagógicos que faciliten que los alumnos adquieran determinadas habilidades o contenidos.

Conocimientos sobre cómo usar la tecnología más adecuada en un marco pedagógico para la impartición de determinada materia.

Fuente: Elaboración propia

Aplicación docente: explicación a modo de plenaria; una vez entendidas las actividades se espera que los niños trabajen de forma individual o colectiva. E seguimiento y retroalimentación docente debe realizarse de forma inmediata y personal.

Tiempo de aplicación: 1 hora clase o 40 minutos diarios.

Recursos: Pizarra digital, Tablet o iPad.

Metodología: Actividades lúdicas 


\section{Aplicaciones a utilizar}

El desarrollo del programa es el siguiente, se trabajan cuatro aplicaciones para IPAD apropiadas para niños de 3 a 6 años indicadas, entre otras cualidades, para la mejora de la motricidad fina. Cada una de las aplicaciones tienen temática diferente. El orden en el que se han empleado las aplicaciones en el programa ha sido metódicamente seleccionado de forma que el programa resultara lo más atractivo y motivador posible para los niños.

\section{Tabla 4}

Aplicaciones tecnológicas a emplearse

\section{Nombre de la} aplicación

\section{Características}

\section{Descripción del uso}


- Ideal para incentivar que los alumnos con dificultades de pronunciación de algunas letras o los alumnos con problemas en la expresión oral puedan mejorar su dicción a base de hablar al dispositivo y escuchar su voz de vuelta.

- Gran amplitud de Aparecen personajes a los cuales los niños movimientos con las deben cortar el pelo, lavar el pelo, peinar o teñir manos. que permiten a los alumnos con menor motricidad seleccionarlos sin problemas. con diferentes utensilios. También pueden colocar al personaje sombreros o pajaritas.

Fuente: Elaboración propia

\section{Tabla 5}

Requerimientos

Requerimientos

Estrategias docentes

Procedimentales

\begin{abstract}
Habilidades cognoscitivas, uso de
dispositivos

conocimientos

tecnología, básicos tecnológicos planificación contenidos e integración de las TIC en el aula.

Manejo de grupos de trabajo, saber hacer, habilidad para integrar las TIC en los planes de clase e interactuar con el material Web de estudio.
\end{abstract}

Actitudinales

Académicos
Capacitación en relación a las TIC páginas Web, integración de los procesos académicos de aprendizaje con las TIC e integración de procedimientos y actitudes relacionados.

\section{Aptitudes de los estudiantes}

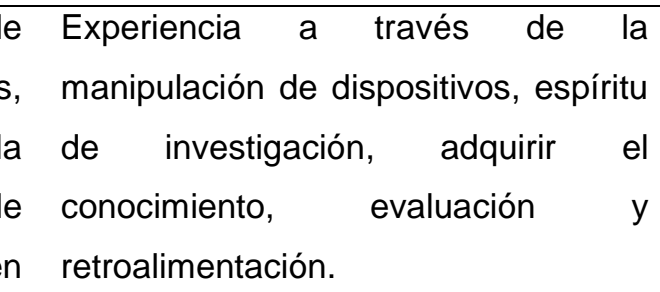
Experiencia a través de la manipulación de dispositivos, espíritu de investigación, adquirir el e conocimiento, evaluación y retroalimentación.

Confianza, creatividad, interacción en el equipo de trabajo, reconocimiento del entorno y conocimiento de las herramientas.

Desarrollo del aprendizaje significativo, integración de las TIC en el conocimiento integral y aplicación de ejercicios para la motricidad fina.

\section{Fuente: Elaboración propia}




\section{Finalidad}

La propuesta no pretende transgredir los principios de praxis del desarrollo motor, sino complementarla. Está orientada a incentivar la formación de representaciones mentales del aprendiz, a través de estímulos apoyados por dispositivos móviles, de manera que él pueda asimilar y procesar dichos elementos, para finalmente transferir y aplicar los conocimientos durante la praxis, permitiéndole, además, autorregular su desempeño motor a través del feedback.

\section{CONCLUSIONES}

Dentro de la discusión del tema es necesario recalcar que se ha llegado a las siguientes conclusiones a partir de las respuestas de los participantes del estudio. Es por ello que con base a la evidencia teórica y conceptual presentada en el apartado de introducción y la evidencia empírica realizada se mencionan las siguientes terminaciones:

Se sostiene que el aprendizaje de la tecnología es parte fundamental dentro del desarrollo integral de individuo, que se refiere a la innovación del conocimiento que va a la par con las competencias que requiere el individuo para el logro de oportunidades ya que desde esta perspectiva se denota que los docentes conceptualizan la importancia de la tecnología, sin embargo, se limitan a utilizarla. Estudios evidencian que la falta de uso de la tecnología en la educación es por falta de conocimientos y de pedagogía en el área de la educación.

Dentro de los hallazgos que tuvo la investigación es que los docentes conocen la importancia de la educación tecnológica en todos los ámbitos, pero no hacen las diferencias en ámbitos específicos, por lo tanto, no conocen sobre el desarrollo de la tecnología aplicado en la psicomotricidad y motricidad fina. La investigación servirá como base para futuras líneas de investigación como: docentes y las causas de su desconocimiento en tecnología, políticas públicas en la docencia y la tecnología, entre otras. 


\section{FINANCIAMIENTO}

No monetario.

\section{AGRADECIMIENTO}

A la Universidad Católica de Cuenca por impulsar el desarrollo de esta investigación.

\section{REFERENCIAS CONSULTADAS}

Adel, J., \& Castañeda, L. (2012). Tecnologías emergentes, ¿pedagogías emergentes? En J. Hernández, M. Pennesi, D. Sobrino, \& A. Vázquez, Tendencias emergentes en educación con TIC (págs. 13-32). Barcelona: Asociación Espiral.

Ardila, A. (2015). Historia y clasificación de las apraxias. Revista Neuropsicología, Neuropsiquiatría y Neurociencias, 15(1), 109-118.

Arufe-Giráldez, V. (2015). La casa del papel. Un proyecto educativo de innovación y gamificación. Universidad de la Coruña.

Bernal-Ávila, E. M., Erazo-Álvarez, J. C., \& Narváez-Zurita, C. I. (2019). Estructuras organizativas favorables a la Eficiencia Empresarial. [Organizational structures favorable to Business Efficiency]. Revista Arbitrada Interdisciplinaria Koinonía, 3-31. doi:http://dx.doi.org/10.35381/r.k.v4i1.370

Berruezo, P. (2008). El contenido de la Psicomotricidad. Revista Interuniversitaria de Formación del Profesorado, 62(22), 19-34.

Cano-García, J. (2018). Aspectos básicos de la gamificación en las aulas de educación infantil. Soria: Universidad de Valladolid.

Cano-García, J. A. (2018). Aspectos básicos de la gamificación en las aulas de educación infantil. Soria: Universidad de Valladolid.

Castillo-Vivas, C. I. (2015). Estrategias que se utilizarán para fortalecer la madurez en el desarrollo psicomotriz de los párvulos del Centro de Educación Inicial "Niños del Nuevo Milenio" de la parroquia Tonsupa. Esmeraldas: Pontificia Universidad Católica del Ecuador.

Da Silva de Goncalves, M. O. (2003). El juego didáctico "carrera en la historia de Venezuela" como estrategia creativa de enseñanza. Valencia (Venezuela): Universidad de Carabobo. 
De Puy, M., \& Miguelena, R. (2017). Importancia de la gamificación en la educación aplicado en entornos de la investigación. 15th LACCEI International MultiConference for Engineering, Education, and Technology, 1-10.

Erazo, J. C., \& Narváez, C. I. (2020). La gestión del capital intelectual y su impacto en la efectividad organizacional de la industria de cuero y calzado en la Provincia de Tungurahua - Ecuador [The management of intellectual capital and its impact on the] Recuperado de: https://n9.cl/52li. Revista Espacios, 254-271.

Ferrer-Planchart, S. (2018). La gamificación como herramienta en el trabajo docente del orientador. Revista Iberoamericana de Educación, 78(1), 165-182.

Ferrer-Planchart, S. (2018). La gamificación como herramienta en el trabajo docente del orientador. Revista Iberoamericana de Educación, 78(1), 165-182.

Gallego, F., Molina, R., \& Llorens, F. (2014). Gamificar una propuesta docente. Diseñando experiencias positivas de aprendizaje. XX Jornadas sobre la Enseñanza Universitaria de la Informática, 1-2.

Jiménez-Torres, A. I., \& García-Lázaro, D. (2015). El proceso de gamificación en el aula. Madrid: Grin Verlag.

Landers, R., \& Auer, E. (2018). Gamification Science, Its History and Future: Definitions and a Research Agenda. Simulation \& Gaming, 1-23.

Mamani-Ticona, R. F. (2017). Significaciones del juego en el desarrollo de la psicomotricidad en educación inicial. La Paz: Universidad Mayor de San Andrés.

Marín-Díaz, V. (2015). La Gamificación educativa. Una alternativa para la enseñanza creativa. DigitalEducation(27), 1-4.

Maya-Zapata, C. T. (2017). El juego como estrategia didáctica para el fortalecimiento del esquema corporal en los niños y niñas del nivel transición del Centro Infantil Casita de Cuentos. El Bagre: Corporación Universitaria Minuto de Dios.

Meneses-Montero, M. (2001). El juego en los niños: enfoque teórico. Revista Educación, 25(2), 113-124.

Muñoz-Oyarce, M. F., \& Almonacid-Fierro, A. (24 de julio de 2017). Cognición, juego y aprendizaje. Infancia, Educación y Aprendizaje (IEYA), 1(1), 162-177.

Núñez-Hernández, B. S., \& Pabón-Fonseca, J. A. (2013). Homo Ludens versus Homo Sapiens en historias de cronopios y de famas. Bucaramanga: Universidad Industrial de Santander. 
Ortíz-Colón, A., Jordán, J., \& Agreda, M. (2018). Gamificación en educación: una panorámica sobre el estado de la cuestión. Educación Pesqui, 44, 1-17.

Quevedo-Álava, R., Corrales-Moreno, L., Palma-Delgado, G., \& Mendoza-Suárez, G. (2020). Psicopedagogía y TIC en período de COVID-19. Una reflexión para el aprendizaje significativo. EPISTEME KOINONIA, 3(5), 202-220. http://dx.doi.org/10.35381/e.k.v3i5.769 